\title{
ARQUITECTURA MODULAR PARA LA GESTIÓN AUTOMÁTICA DEL TRÁFICO EN ROTONDAS
}

\author{
Adrian Lara-Dominguez \\ Escuela Técnica Superior de Ingeniería del Diseño, Universitat Politècnica de València (UPV), \\ Valencia, España, adrianfagot@gmail.com \\ Pedro Uribe-Chavert \\ Escuela de Doctorado, Universitat Politècnica de València (UPV), \\ Valencia, España, pedurcha@doctor.upv.es \\ Jose-Luis Poza-Lujan \\ Instituto de Automática e Informática Industrial (ai2), Universitat Politècnica de València (UPV), \\ Valencia, España, jopolu@upv.es \\ Juan-Luis Posadas-Yagüe \\ Instituto de Automática e Informática Industrial (ai2), Universitat Politècnica de València (UPV), \\ Valencia, España, jposadas@upv.es
}

\section{Resumen}

En los entornos de movilidad, calles o carreteras, los cruces y las rotondas pueden generar problemas de atascos. Poder optimizar el tráfico entrante y saliente de un cruce o rotonda es uno de los campos de investigación de los sistemas de control inteligente. Para optimizar el tráfico se debe disponer de dispositivos capaces de detectar los vehículos así como de actuar, regulando el tráfico, de forma dinámica para adaptarse a las distintas circunstancias. El sistema presentado busca la adaptación a las necesidades de tráfico en una rotonda. Dependiendo de la saturación de cada carril de entrada se intenta crear un tráfico fluido y continuo en el interior de la misma. Para lograr mejorar el tráfico, en este trabajo se presenta una arquitectura modular que permite adaptarse a cualquier cruce o rotonda para, a partir del control específico de un sector, mejorar el rendimiento global. El sistema simulado está compuesto por dispositivos independientes, que, dependiendo de la información adquirida varían el tiempo de paso. Se presenta, asimismo, un experimento de simulación en el que se pone en valor la capacidad de reducir el tráfico adaptando los tiempos de paso en función de la demanda. Los resultados muestran que es posible descongestionar una rotonda cuando se automatizan dinámicamente los tiempos de paso sobre los que se tiene control.

Palabras clave: Control inteligente, Control distribuido, Gestión del tráfico, Ciudades inteligentes

\section{Introducción}

El concepto de Industria 4.0 (I4.0) se centra en lograr un sistema eficiente en entornos automatizados y con un alto nivel de inteligencia [11]. Para ello, el modelo I4.0 se caracteriza principalmente por aspectos como [5]:

- Adaptación al cambio, al tener periodos cortos en los que se incorporan, o modifican, tanto los productos como los propios componentes del sistema.

- Demanda personalizada, que se plasma en una adaptación de la producción en la industria, o de la acción de control, a las circunstancias concretas en cada momento.

- Flexibilidad, que permite una respuesta temprana, lo más rápida posible, a las demandas de los usuarios.

- Descentralización, tanto en la obtención de los datos como en la toma de decisiones. Esta descentralización se debe centrar en lograr una agilidad que cumpla la siguiente característica.

- Eficiencia en el manejo de los recursos. Esta eficiencia no sólo está asociada a los costos, sino también enfocada al cambio social y a la sostenibilidad ambiental.

Consecuentemente, las arquitecturas que sirven de modelo a los sistemas deberán cubrir las carac- 
terísticas anteriores. Estas arquitecturas deben, además, orientarse hacia la optimización de un sistema de control inteligente distribuido [1]. Esta optimización implica, consecuentemente, tener que orientar el sistema hacia una computación cercana al dispositivo o un sistema inteligente distribuido del Internet of Things [2] Para este tipo de sistemas es posible adaptar modelos de arquitecturas basadas en el 'Edge', 'Fog' y 'Cloud' [7].

En el caso del trabajo presentado, se centra en dispositivos que se encuentran en contacto con el entorno físico real, es decir se centra en el 'Edge'. En [10] se define el 'Edge' como todo dispositivo intermediario entre la realidad y la nube. Es decir el Edge está definido en función de la red.

La computación en el 'Edge' [6] propone trasladar la capacidad computacional de los servidores en la nube, generalmente centralizados, a los nodos periféricos cercanos al usuario o al entorno real. La informática del 'Edge' proporciona dos mejoras importantes a las que proporciona la computación en la nube. El primero es que los nodos del 'Edge' pueden preprocesar grandes cantidades de datos antes de transferirlos a los servidores centrales en la nube. El otro es que los recursos de la nube se optimizan habilitando nodos de control en el 'Edge' que ya tienen una capacidad de computación. En resumen, un control, con un cierto nivel de inteligencia, al nivel del 'Edge', permite reducir la carga de comunicaciones y de computación en la nube, además de de responder de una forma más ágil a cambios de contexto.

Los entornos de ciudades inteligentes necesitan de arquitecturas adaptadas a las necesidades de cada espacio [4]. Basándose en el espacio común en el que interaccionan varios dispositivos, en [9] se define el 'Edge' como la zona donde el rango de operación de dichos dispositivos. Basándose en las premisas anteriores, en este artículo se presenta una arquitectura modular de control inteligente distribuido que es posible ubicar en el modelo de arquitecturas de industria 4.0 y que cumple el paradigma de computación en Cloud/Fog/Edge. La arquitectura dispone de elementos modulares en los que situar elementos de computación. Para experimentar, se ha implementado en MatLab con Simulink un modelo de tráfico básico de entrada a la rotonda.

En la siguiente sección se muestran los detalles generales de la arquitectura del sistema modular de control distribuido. Seguidamente se modela el caso de estudio realizado para, finalmente, exponer la experimentación realizada con una sección de rotonda, y los resultados de aplicar un control adaptativo en comparación con el control de tiempos fijos.

\section{Arquitectura del sistema}

El control inteligente del tráfico tiene una gran dependencia de los modelos de implementación física utilizados, lo cual implica una gran dependencia de la disponibilidad y ubicación de los sensores, controladores y actuadores. Diferentes arquitecturas afrontan la implementación de los sistemas de control distribuido basándose, la mayoría de los trabajos, en una organización centralizada o en agrupaciones semi-centralizadas. En cualquiera de los casos, lo habitual es que el sistema dependa de un nodo supervisor que gestiona a una serie de nodos de control dependientes [8]. Estos nodos de control generan, y consumen, datos. La gestión de estos datos es habitual que se delegue en servidores dedicados, conectados en la capa de la nube. Estos servidores son los responsables de recibir los datos, mantenerlos para proporcionar un histórico de datos, u ofrecerlos para que diferentes elementos del sistema puedan emplearlos en sus algoritmos [3].

En el presente artículo, se plantea una arquitectura modular mostrada en la figura 1 .

Esta arquitectura implementa un control descentralizado y modular. El elemento básico de la arquitectura es el módulo de control (CM) definidos como nodos que disponen de sensores y actuadores, junto con un control reactivo. Un ejemplo del módulo de control es un detector de vehículos o una de las luces del semáforo. La unión de varios CM, mediante un canal de comunicación, es un Recurso Inteligente (del inglés, SR). Por ejemplo, un semáforo inteligente. Un SR puede estar formado por diferentes tipos de CM, pudiendo haber diferentes $\mathrm{SR}$ en una misma rotonda. El área de interacción de los distintos CM de un SR se llama Área de Operación (OA). El área de comunicación entre distintos SR se llama Área de Control (CA). Por ejemplo, el área de control para las comunicaciones entre varios semáforos en una misma rotonda. A su vez, CM y SR pueden interactuar con servicios en la nube.

El hecho de que un elemento tan cercano como un módulo de control pueda llegar a alcanzar directamente a un servidor en la nube, facilita la compartición de datos ya procesados con otras áreas de control. En el caso del entorno de experimentación, presentado más adelante, un Recurso Inteligente es un semáforo que dispone de módulos de control independientes para detectar vehículos, detectar peatones, señalizar el paso a vehículos y a peatones, e incluso iluminar el espacio de espera de los peatones en función de la detección de los mismos. 


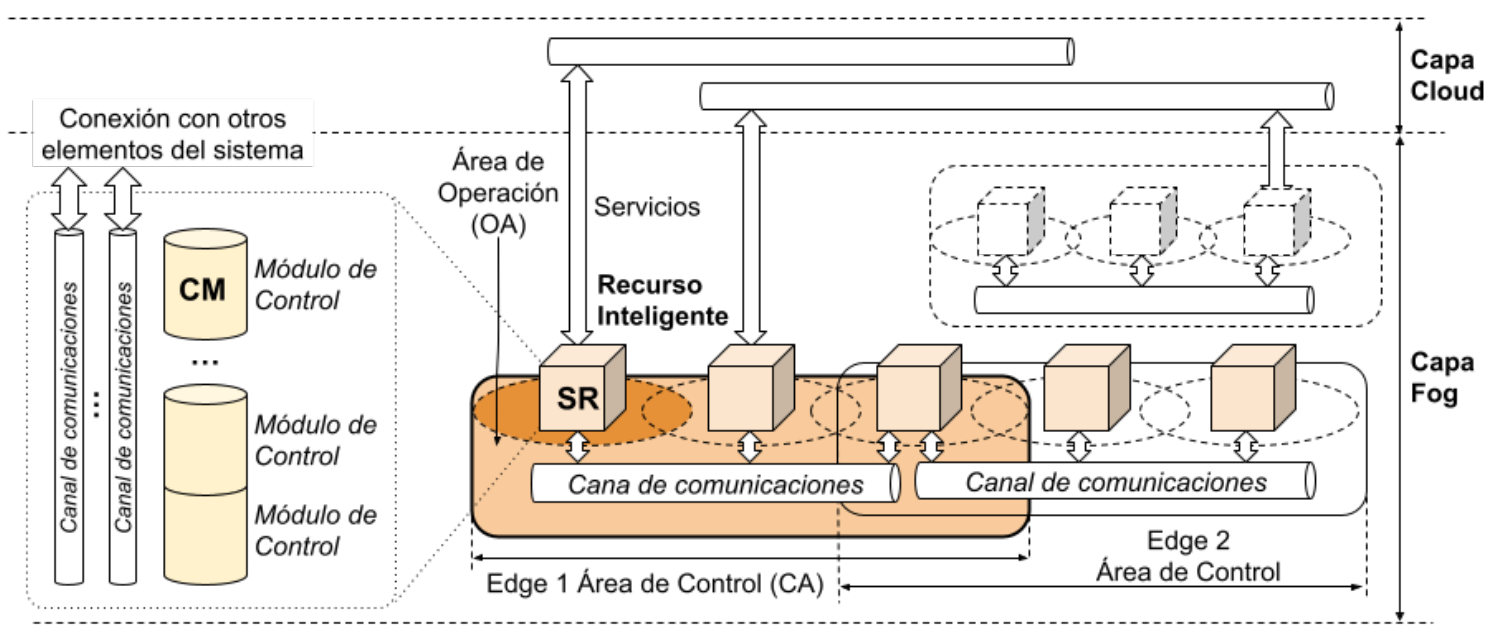

Figura 1: Arquitectura propuesta basada en el modelo (cloud/fog). Los módulos de control se combinan para formar un recurso inteligente que, a su vez, al comunicarse con otros recursos inteligentes forman un área de control. Este área se caracteriza por tener una comunicación muy cercana entre los recursos, sin necesidad de esperar acciones de control desde la nube.

- Mecánica $\rightarrow$ Diseño modular del módulo donde irá incorporado el semáforo

- Electrónica $\rightarrow$ Conectado con el módulo maestro (base) vía bus I2C

- Computación $\rightarrow$ Se enciende y se apaga respecto lo que dictamine el módulo Maestro

- Mecánica $\rightarrow$ Diseño modular del módulo donde irá incorporado el semáforo

- Electrónica $\rightarrow$ Conectado con el módulo maestro (base) vía bus I2C

- Computación $\rightarrow$ Se enciende y se apaga respecto lo que dictamine el módulo Maestro

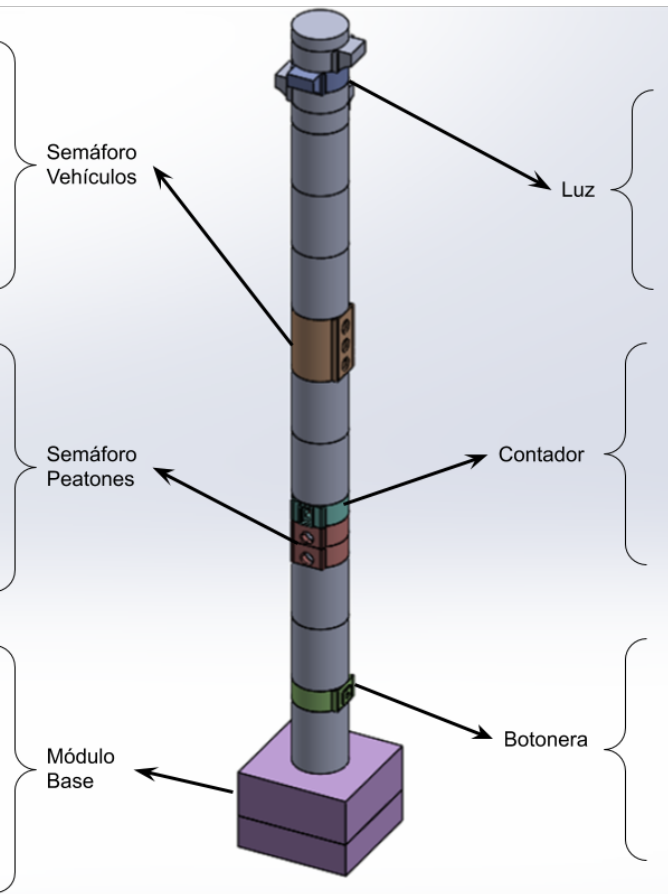

Mecánica $\rightarrow$ Diseño modular del módulo donde irá incorporado la luz

- Electrónica $\rightarrow$ Conectado con el módulo maestro (base) vía bus I2C

- Computación $\rightarrow$ Se enciende y se apaga

- Mecánica $\rightarrow$ Diseño modular del módulo donde irá incorporado la luz

- Electrónica $\rightarrow$ Conectado con el módulo maestro (base) vía bus I2C

- Computación $\rightarrow$ Indica los segundos que le quedan a los peatones para cruzar

- Mecánica $\rightarrow$ Diseño modular del módulo donde irá incorporado la luz

- Electrónica $\rightarrow$ Conectado con el módulo maestro (base) vía bus $\mathrm{I} 2 \mathrm{C}$

- Computación $\rightarrow$ Al pulsarlo, avisa al módulo maestro de que el peatón desea cruzar

Figura 2: Ejemplo de un Recurso Inteligente. En este caso, se dispone de elementos de detección y de actuación que juntos pueden seleccionar un control adaptativo a las circunstancias que estos mismos Recursos Inteligentes detectan. Por ejemplo, con un flujo mayor de vehículos se puede ampliar el tiempo de paso de los mismos para salir de la rotonda y descongestionarla, o disminuir dicho tiempo para evitar introducir más vehículos en la rotonda de los que ésta pueda asumir. 


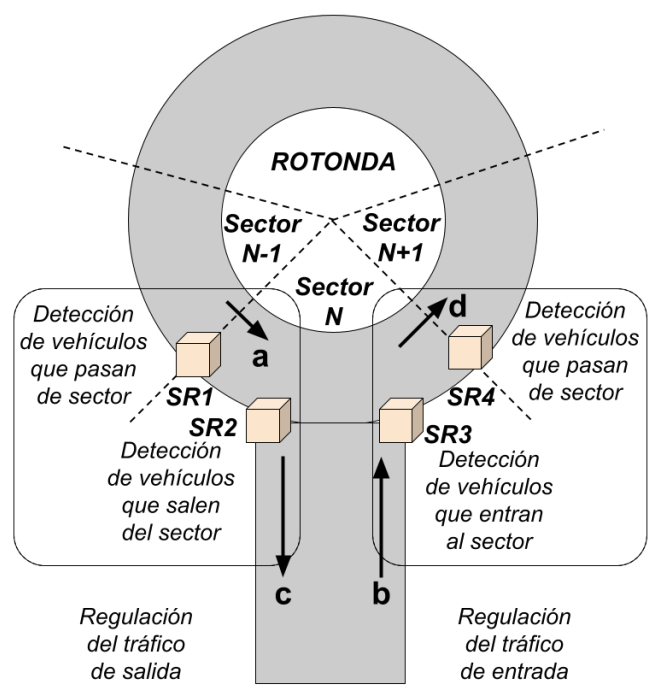

Figura 3: Sector de rotonda y posibles configuraciones dependiendo del tipo de calle conectada a la rotonda.

\section{Modelado y control implementado}

\subsection{Rotonda como conjunto de sectores}

Se ha decidido realizar la simulación, diseño y control de una sección de rotonda basada en calle por la modularidad que ello representa y la escalabilidad que tiene dicho sistema, al poder crear mediante la unión de varias secciones una rotonda entera, lo que ayuda al diseño de estas y su ejecución en ciudades y urbes. Las secciones de rotonda pueden detectar los coches que salen de ellas y comunicarse con el resto de secciones, estos métodos de detección y comunicación no son parte de este estudio pero pueden desarrollarse posteriormente e incorporarse al implantar el modelo en una situación real.

Para facilitar, tanto el modelado como la simulación, una rotonda se divide en secciones. Cada sección de rotonda se puede considerar como un enlace entre la calle y la rotonda (figura 3). En cada sección "n" con su calle asociada se distingue entre el tráfico "a" proveniente de la sección "n1 ", el tráfico "c" que sale de la rotonda a través de la calle, el tráfico "b" que entra en la rotonda a través de la calle y el tráfico "d" que pasa a la sección "n+1". Los dispositivos que se incluyan en la rotonda deben ser capaces de detectar tanto el tráfico en ésta (a y d) como el tráfico entrante y saliente (b y c respectivamente). Además, también es conveniente que los dispositivos puedan regular el tráfico.

Este modelado tiene como principal ventaja el he-
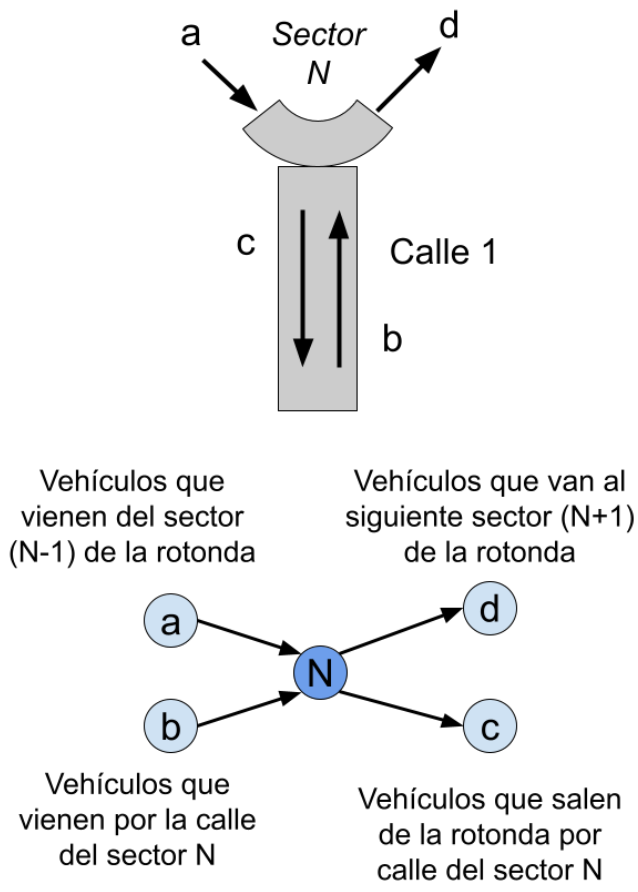

Figura 4: Sector de rotonda y posibles configuraciones dependiendo del tipo de calle conectada a la rotonda.

cho de aislarse del tipo de calle que aporta o recibe vehículos en la rotonda. Las calles que sólo reciben vehículos de la rotonda no tienen componente $b, \mathrm{y}$ las calles que sólo aportan vehículos a la rotonda no tienen componente $c$. Además, tal como se comentó en el apartado de arquitectura, cada sector de rotonda puede considerarse como un conjunto de recursos del 'Edge', por lo que el control de la misma se ve facilitado. Básicamente, un sector de rotonda se podría llegar a modelar como un sistema de control donde las entradas a y b, determinan la carga entrante del sector, y las salidas c y d permiten descargar el mismo (figura 4).

\subsection{Algoritmo de control}

Tal y como se puede contemplar en el diagrama de flujo de la figura 5 , el sistema consta de un tiempo de ciclo constante en el tiempo. Este tiempo puede ser modificable para distintas simulaciones. Este tiempo es la suma del tiempo en rojo más el tiempo en verde. Estos tiempos varían dependiendo de la longitud de cola que tenga la entrada a la rotonda.

El control comienza con el semáforo de la calle de entrada a la rotonda en rojo para vehículos. El módulo de computación calcula la longitud de cola colab de la calle de entrada. Si colab es mayor o igual a un umbral colab $b_{t o p}, 7$ en el caso del ejem- 


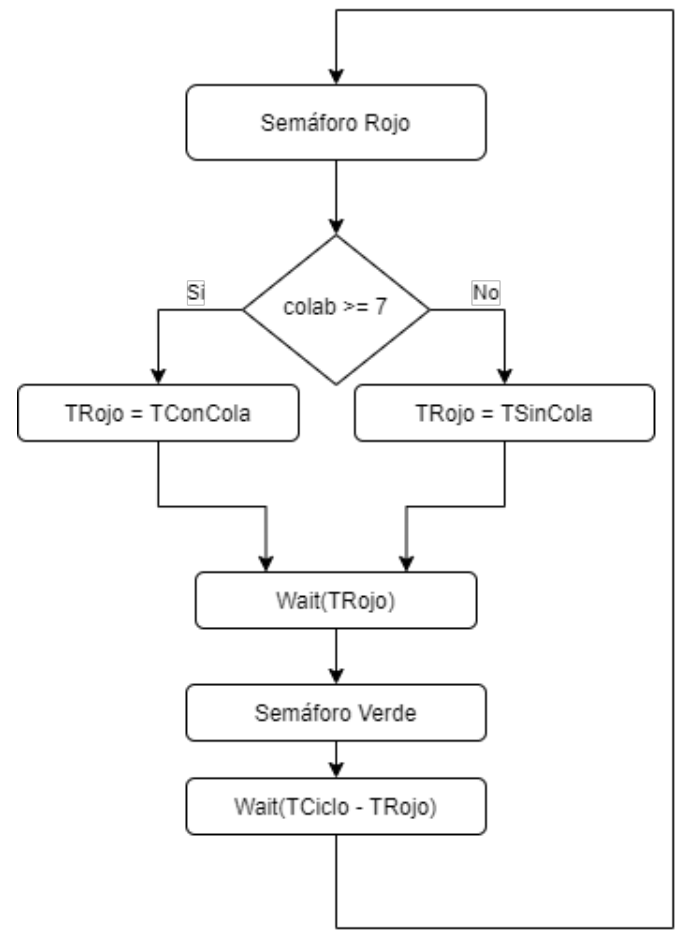

Figura 5: Diagrama de flujo del control del semáforo

plo, el tiempo de espera en rojo se determina a un valor máximo para descargar la rotonda o tiempo con cola TConCola. En cambio, si colab es menor al umbral, el tiempo de espera en rojo se asigna al valor mínimo para descargar la calle o TSinCola. Los tiempos TConCola y TSinCola tienen una diferencia variable dependiendo de la carga que se haya medido en el sector de la rotonda. A continuación, se espera el correspondiente tiempo calculado anteriormente y se pone el semáforo en verde, estando un tiempo igual al total de ciclo menos el tiempo en rojo calculado. El tiempo de ciclo se ha determinado fijo en el caso de los experimentos que se presentarán más adelante. Con esto se consigue que, cuanto se genere cierta saturación en la entrada de la rotonda, se consiga despejar más rápidamente.

\section{Experimentos y resultados}

A partir de la arquitectura y el caso de estudio expuesto anteriormente, para comprobar la conveniencia de emplear un algoritmo de control adaptativo, se van a medir las siguientes variables:

- at: Número total de vehículos entrantes por "a" detectados.

- parac: Número total de vehículos que van a salir por la calle.
Tabla 1: Resultados de las variables medidas con el control en tiempos fijo y el control de tiempo variable.

$\begin{array}{lrr}\text { Variables } & \text { Tiempo fijo } & \text { Tiempo variable } \\ \text { at } & 272 & 272 \\ \text { parac } & 136 & 136 \\ \text { parad } & 136 & 136 \\ \text { bt } & 179 & 218 \\ \text { ct } & 136 & 136 \\ \text { d1t } & 135 & 135 \\ \text { d2t } & 178 & 217 \\ \text { dt } & 313 & 352 \\ \text { colab } & 47 & 8 \\ \text { cs } & 2 & 2\end{array}$

- parad: Número total de vehículos que van a salir por el siguiente tramo y provienen de la anterior sección.

- bt: Número total de vehículos entrantes provenientes de la calle.

- ct: Número total de vehículos salientes hacia la calle.

- d1t: Número total de vehículos salientes hacia el siguiente tramo provenientes del anterior tramo.

- d2t: Número total de vehículos salientes hacia el siguiente tramo provenientes de la calle de entrada.

- dt: Número total de vehículos que se dirigen a la siguiente sección.

- colab: Cola generada en la calle de entrada.

- cs: Número de vehículos en la sección.

La tabla 1 muestra los resultados tras 1200 segundos de simulación tanto para el sistema controlado por tiempos fijos, como para el controlado por tiempos variables.

En la columna "Tiempo fijo" de la tabla 1 se puede observar que el sistema de distribución de vehículos al 50\% para que estos se dirijan a "c" o a "d" funciona, ya que se incorporan por "a" 272 transeúntes y se distribuyen 136 equitativamente hacia cada salida. El problema que se ve reflejado es la gran cola que se produce con los parámetros establecidos, tras 20 minutos de simulación se genera una cola de 47 vehículos en la calle, lo que supone un colapso grande en el tráfico.

Cuando el algoritmo de control empleado es el de tiempos variables, se producen algunos cambios interesantes. Los resultados de emplear tiempo 


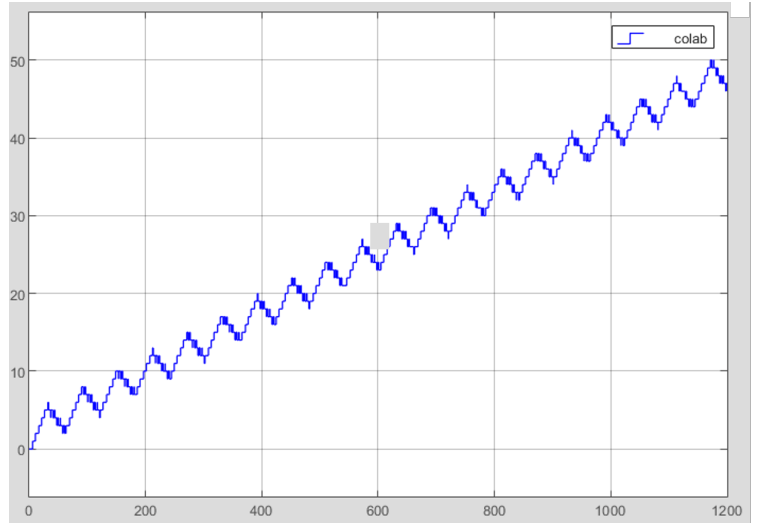

Figura 6: Evolución de la cola de vehículos a la entrada de la rotonda con un control por tiempos fijos y simulación de 1200 segundos

variable se muestra en la columna "Tiempo variable" de la tabla 1. En este caso los tiempos de entrada de vehículos y características del sistema son iguales que en los datos anteriores y el tiempo de simulación se mantiene en 1200 segundos. En los resultados se puede observar que los vehículos de entrada desde la anterior sección son los mismos, pero los vehículos incorporados desde la calle han aumentado, esto debido a que por el control se permite un mayor tiempo de paso para los vehículos que se incorporan a la rotonda. El dato más interesante es el de cola que ha quedado establecido tras la simulación en 8 vehículos mucho menor que en los datos obtenidos con el control de tiempos fijos.

Se han obtenido también mediante la herramienta scope que facilita Simulink, la progresión del número de vehículos en sección y número de vehículos en cola a lo largo de la simulación. En la figura 6 se muestra la evolución de la cola de vehículos a lo largo de la simulación con el algoritmo de tiempos fijos. En el eje $x$ se muestra la variable tiempo y en el eje $y$ se muestra la longitud de la cola de entrada a la rotonda.

Cómo se puede observar, la primera gráfica muestra un perfil serrado donde las rampas de subida corresponden al tiempo en que el semáforo está cortando el paso y las de bajada a cuando el semáforo deja paso. Es una gráfica ascendente debido a que este tipo de control con estas condiciones no logra descongestionar el tráfico de la calle, lo que genera cola y acumulación de vehículos. El pico más alto de vehículos se muestra en 50 acumulados. En lo que respecta a carga en el sector de la rotonda, en la simulación, se obtiene que el número de vehículos en la sección no supera los 3 vehículos circulando por ella, lo que muestra que no se ha saturado en ningún momento (figura 7 .

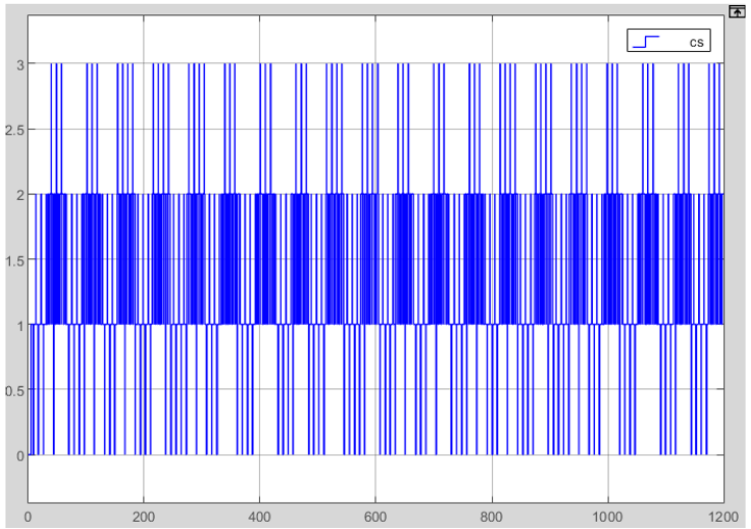

Figura 7: Evolución de la carga de vehículos en el sector de la rotonda con un control por tiempos fijos y simulación de 1200 segundos

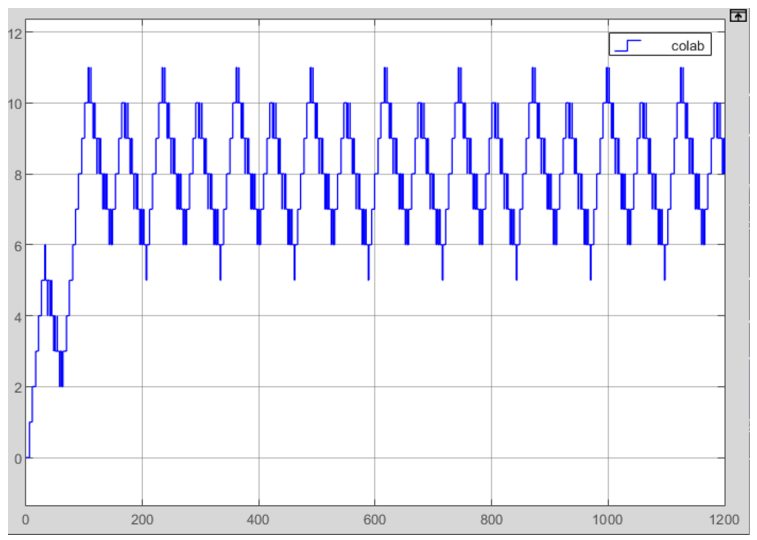

Figura 8: Evolución de la cola de vehículos a la entrada de la rotonda con un control por tiempos variables simulación de 1200 segundos

En la figura 8 se muestra la evolución de la cola de vehículos a lo largo de la simulación con el algoritmo de tiempos variables. Se puede observar, al igual que en el anterior caso, que también se forma un perfil serrado.

Sin embargo, en este caso, al entrar en funcionamiento cuando se detecta una cola mayor de 7 vehículos, el segundo modo del semáforo el cual corta el paso durante 20 segundos y deja pasar vehículos cada 40, con este control, se puede observar una mejoría en cuanto a la cola generada, ya que al dejar más tiempo de paso para los vehículos que se incorporan de la calle cuando el sistema detecta cierto tamaño de cola. Consecuentemente hace que la congestión de tráfico disminuya y que la máxima cola generada bajo estas condiciones sea de 11 vehículos. Al igual que en el caso de tiempos fijos, el total de vehículos en la sección de la rotonda, no supera en ningún momento los 3 vehículos (Figura 9.

Este último aspecto, el de la carga de vehículos 
XLII Jornadas de Automática

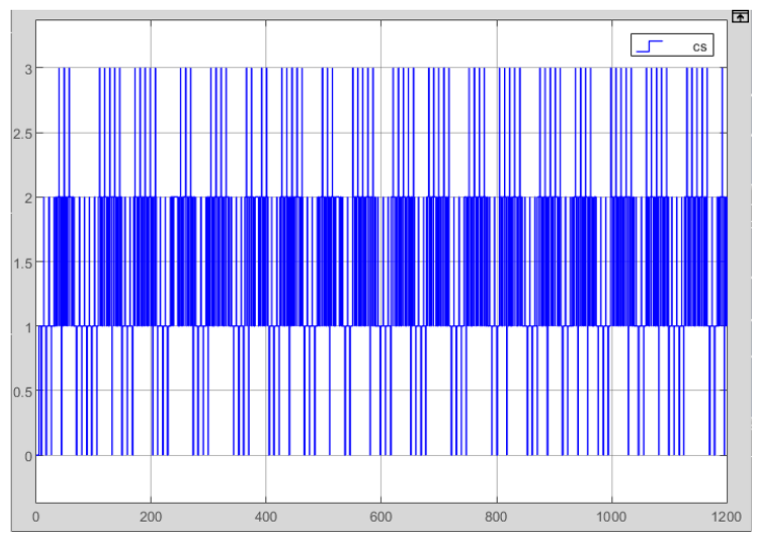

Figura 9: Evolución de la carga de vehículos en el sector de la rotonda con un control por tiempos variables y simulación de 1200 segundos

en el sector, es importante debido a que la capacidad de vehículos se estableció en 3. Consecuentemente, en ambos casos la rotonda está ofreciendo un servicio similar al tráfico. Sin embargo, en el segundo de los casos las colas de espera son menores.

\section{Conclusiones}

Actualmente el control modificando los tiempos de los estados de los semáforos en rotondas no está muy extendido. A su vez el crear un diseño de rotonda modular genera una escalabilidad muy grande y permite la adaptación de este sistema a cualquier tipo de rotonda ya existente de manera rápida y eficiente, ya que la aplicación del control por cola, mejoraría el estado del tráfico.

Gracias al control diseñado y la mejora de tráfico que conlleva su implementación se consigue reducir la contaminación producida en retenciones por los vehículos parados en estos

Optimizando el tráfico se consigue que el tiempo empleado para realizar un desplazamiento sea menor, lo que conlleva que los conductores de vehículos pierdan menos tiempo en sus trayectos. Por otra parte, gracias a un buen control del tráfico el número de accidentes se ve reducido. Ambas mejoras ayudan a mejorar la calidad de vida de las ciudades. A partir del trabajo desarrollado en este proyecto se puede trabajar en la implantación de nuevos sistemas de control y adaptar el sistema a las necesidades de cada rotonda al tratarse de un sistema modular. Además, pueden simularse los problemas de retenciones que podrían surgir en una futura rotonda que se desee instalar, ajustando los parámetros, y optimizar al máximo el tráfico en esta, haciendo uso de la herramienta de simulación con diferentes cambios en
Computadores y Control

el control. Una de las ampliaciones posibles sería el desarrollo de un prototipo del sistema diseñado, la cual no ha sido posible realizar debido a la actual situación de pandemia y las restricciones de acceso a los laboratorios que esta ha desarrollado.

\section{Agradecimientos}

Trabajo apoyado por el Ministerio de Ciencia e Innovación de España MICINN: Proyecto CICYT PRECON-I4: "Sistemas informáticos predecibles y confiables para la Industria 4.0" TIN2017-86520C3-1-R.

\section{English summary}

\section{INTELLIGENT CONTROL IN THE EDGE AND THE FOG: AUTOMATIC TRAFFIC MAN- AGEMENT IN ROUNDABOUTS}

\section{AbstractCrossroads and roundabouts,}

can produce traffic jams if traffic management is not optimized. To manage these elements, it is necessary to have devices able to detect the level of traffic as well as to act dynamically in order to adapt the cross access time in each street. The system seeks to adapt to the traffic needs in the roundabout, depending on the saturation of each entrance lane, trying to create a fluid and continuous traffic inside it. In order to optimize the traffic, in this work, a simulation experiment is presented in which the ability to reduce traffic is valued by adapting the access times to a roundabout according to the traffic demand. The simulated system is composed of independent devices, which, depending on the information acquired, can change the access time to the roundabout. The results show that it is possible to reduce the traffic load in a roundabout when access times are dynamically automated.

Keywords: Intelligent control, Distributed control, Traffic management, Smart cities. 


\section{Referencias}

[1] Andoni Amurrio, Ekain Azketa, J Javier Gutierrez, Mario Aldea, and Jorge Parra. A review on optimization techniques for the deployment and scheduling of distributed real-time systems. Revista Iberoamericana de Automática e Informática Industrial, 16(3):249-263, 2019.

[2] PJ Escamilla-Ambrosio, A Rodríguez-Mota, E Aguirre-Anaya, R Acosta-Bermejo, and M Salinas-Rosales. Distributing computing in the internet of things: cloud, fog and edge computing overview. In NEO 2016, pages 87115. Springer, 2018.

[3] Hung-Chin Jang and Ting-Kuan Lin. Trafficaware traffic signal control framework based on sdn and cloud-fog computing. In 2018 IEEE 88th Vehicular Technology Conference (VTC-Fall), pages 1-5. IEEE, 2018.

[4] Rida Khatoun and Sherali Zeadally. Smart cities: concepts, architectures, research opportunities. Communications of the $A C M$, $59(8): 46-57,2016$.

[5] Heiner Lasi, Peter Fettke, Hans-Georg Kemper, Thomas Feld, and Michael Hoffmann. Industry 4.0. Business \& information systems engineering, 6(4):239-242, 2014.

[6] He Li, Kaoru Ota, and Mianxiong Dong. Learning iot in edge: Deep learning for the internet of things with edge computing. IEEE network, 32(1):96-101, 2018.

[7] Jie Lin, Wei Yu, Nan Zhang, Xinyu Yang, Hanlin Zhang, and Wei Zhao. A survey on internet of things: Architecture, enabling technologies, security and privacy, and applications. IEEE Internet of Things Journal, 4(5):1125-1142, 2017.

[8] Mohsen Mahoor, Farzad Rajaei Salmasi, and Tooraj Abbasian Najafabadi. A hierarchical smart street lighting system with brute-force energy optimization. IEEE Sensors Journal, 17(9):2871-2879, 2017.

[9] Jose-Luis Poza-Lujan, Juan-Luis PosadasYagüe, José-Enrique Simó-Ten, and Francisco Blanes. Distributed architecture to integrate sensor information: Object recognition for smart cities. Sensors, 20(1):112, 2020.

[10] Weisong Shi, Jie Cao, Quan Zhang, Youhuizi $\mathrm{Li}$, and Lanyu Xu. Edge computing: Vision and challenges. IEEE internet of things journal, 3(5):637-646, 2016.
[11] František Zezulka, Petr Marcon, I Vesely, and Ondrej Sajdl. Industry 4.0-an introduction in the phenomenon. IFAC-PapersOnLine, 49(25):8-12, 2016.

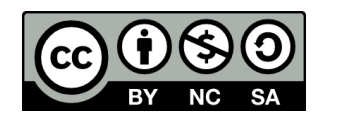
(C) 2021 by the authors. Submitted for possible open access publication under the terms and conditions of the Creative Commons Attribution CC BY-NC-SA 4.0 license (https://creativecommons.org/licenses/by-ncsa/4.0/deed.es). 